\title{
Minorités et circulations techniques : la confection des armures à Tours (XVe-XVIe siècles)
}

Minorities and dissemination of skills: the making of armours in Tours

(xvth and xvith centuries)

\section{Eric Reppel}

\section{OpenEdition}

\section{Journals}

Édition électronique

URL : http://journals.openedition.org/dht/957

DOI : 10.4000/dht.957

ISSN : 1775-4194

\section{Éditeur :}

Centre d'histoire des techniques et de l'environnement du Cnam (CDHTE-Cnam), Société des élèves du CDHTE-Cnam

\section{Édition imprimée}

Date de publication : 1 juin 2008

Pagination : 24-31

ISBN : $978-2-95-30779-1-9$

ISSN : 0417-8726

\section{Référence électronique}

Eric Reppel, « Minorités et circulations techniques : la confection des armures à Tours (XVe-XVle siècles) ", Documents pour I'histoire des techniques [En ligne], 15 | $7^{\text {er }}$ semestre 2008, mis en ligne le 08 novembre 2010, consulté le 07 septembre 2020. URL : http://journals.openedition.org/dht/957 ; DOI : https://doi.org/10.4000/dht.957 


\title{
Minorités et circulations techniques: la confection des armures à Tours $\left(x v^{e}-x v I^{e}\right.$ siècles)
}

\author{
Éric Reppel, \\ Centre d'études supérieures \\ de la Renaissance (Tours)
}

\begin{abstract}
Résumé
Aux $x v^{e}$ et $x v I^{e}$ siècles, Tours apparaît comme un des centres armuriers les plus importants du royaume de France. La présence, à Tours, tout au long de ces deux siècles, d'une importante communauté d'armuriers d'origine étrangère, principalement originaires du nord de la péninsule italienne mais également des Flandres et des terres d'Empire, n'est pas étrangère à ce développement et à cette réputation d'excellence qu'ont acquis les armures fabriquées dans la cité ligérienne. L'immigration de ces artisans spécialisés, issus de dynasties d'armuriers déjà célèbres, a été encouragée par une volonté politique délibérée des souverains français afin de favoriser la diffusion dans le royaume d'un savoir-faire unique. Installés en Touraine, ces armuriers transalpins, allemands ou encore flamands ont bénéficié de statuts privilégiés et de nombreux avantages. Fournisseurs des rois et des grands personnages du royaume, ils ont su s'intégrer parfaitement au reste de la communauté, et ont contribué, par le biais de leurs apprentis, à pérenniser l'art dont ils étaient dépositaires.
\end{abstract}

Résumés et mots clés en anglais sont regroupés en fin de volume, accompagnés des mots clés français.

ors de son passage à Tours en octobre 1517, AntoLnio De Béatis, secrétaire du cardinal Louis d'Aragon, ne retient qu'une chose de la cité ligérienne : il note dans son journal de voyage que les épées qu'on y fabrique dans les quartiers bruyants des bords de Loire sont de très belle facture et d'une qualité exceptionnelle.

Il est vrai qu'aux xv et $x v{ }^{e}$ siècles, Tours apparaît comme un des centres armuriers les plus importants du royaume de France ${ }^{1}$. De plus, il est possible de constater la présence, à Tours, tout au long de ces deux siècles, d'une importante communauté d'armuriers d'origine étrangère. Si les archives tourangelles se sont révélées

\footnotetext{
1 La production d'armement défensif a donné naissance à des spécialités bien distinctes et, sous le terme générique d'armurier, sont confondus aujourd'hui des artisans qui, au xvie siècle, exerçaient des métiers différents, avec leurs propres types de production d'armement défensif: armuriers, brigandiniers, haubergeonniers, fourbisseurs de harnois ou encore sommeliers d'armures.
}

peu prolixes sur ces informations, I'utilisation d'autres sources d'information, telles que les actes de naturalisation conservés aux Archives nationales et certains ouvrages tirés de la bibliographie consacrée à la question de l'armurerie, ont permis de dresser une liste nominative des artisans allogènes exerçant dans la cité$^{2}$. Les patronymes permettent aussi de cerner ces armuriers étrangers, dans la mesure où certains noms font référence à une localité ou ont une consonance révélatrice du pays d'origine. Toutefois, une difficulté supplémentaire intervient dans la mesure où une grande majorité de ces noms ont été francisés rendant I'identification malaisée. C'est pourquoi cette liste ne saurait être non plus être exhaustive. Toutefois, il a été possible de dénombrer à Tours trente-sept armuriers en activité pour le $x v^{e}$ siècle ${ }^{3}$ et vingt-deux individus pour

\footnotetext{
2 Voir document 4.

3 Painsonneau S., "L'armurerie tourangelle au $x v^{e}$ siècle : la fabrication et le commerce des armures », mémoire de maîtrise de
} 


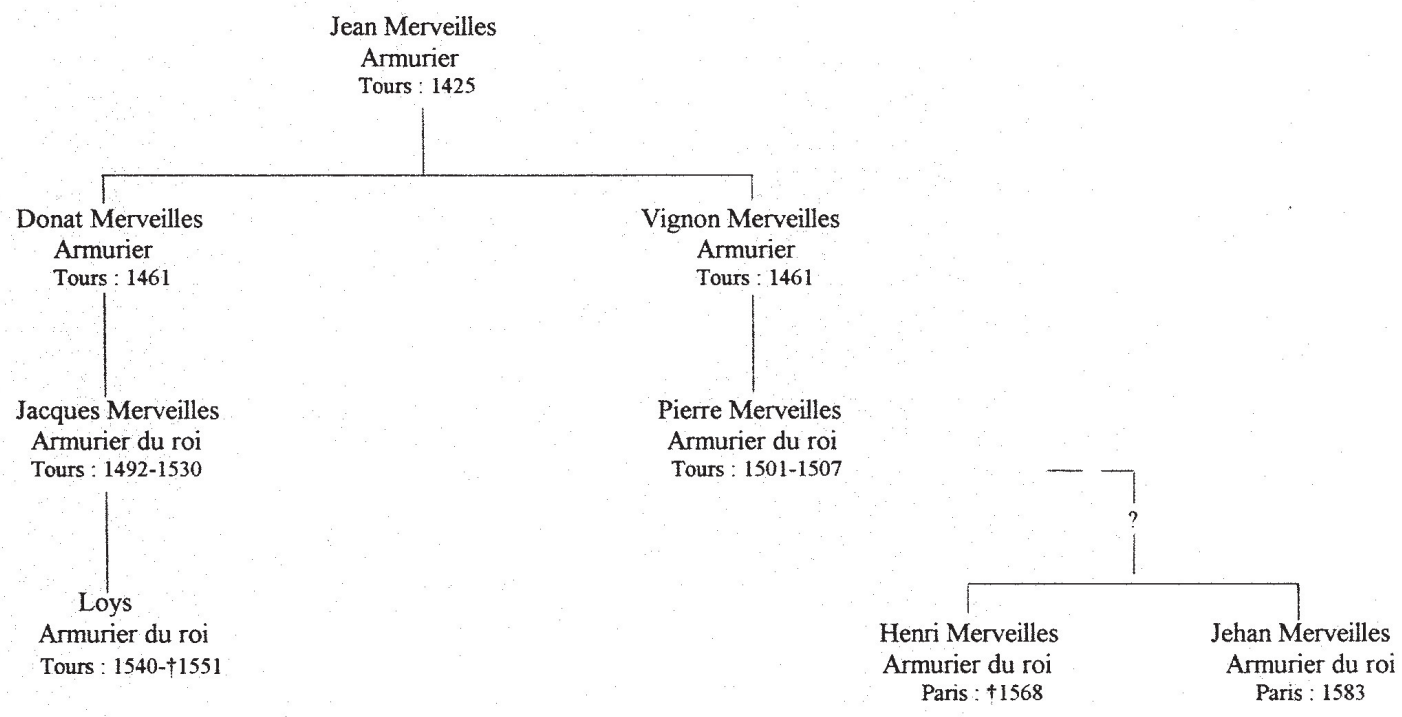

I'ensemble du $x v I^{e}$ siècle qui viennent d'au-delà des frontières du royaume ${ }^{4}$.

Ces armuriers étrangers, principalement originaires du nord de la péninsule italienne ont été des acteurs majeurs de cette réputation d'excellence qu'ont acquises les armures tourangelles ${ }^{5}$. En effet, toujours selon ces sources, il est possible de constater que plus les procédés techniques à mettre en œuvre sont nouveaux, plus la proportion d'étrangers compétents est importante. Ce qui est le cas pour l'armurerie, activité qui demande un haut degré de technicité. Ce regroupement important d'armuriers transalpins n'est cependant pas spécifique à Tours. Matthias Pfaffenbichler, conservateur au Leib-Rüstkammer de Vienne constate que les plus importantes communautés du royaume se sont établies à Bordeaux et surtout à Lyon, I'une des principales places armurières du royaume $e^{6}$.

Se pose alors la question de l'intégration de ces individus tant sur le plan local qu'à l'échelle du royaume et sur les apports techniques qu'ils ont apportés à l'art de l'armurerie défensive. Grâce à l'ensemble des informations recueillies, il est possible de répondre à cette

I'Université de Tours, sous la direction de Joëlle Burnouf, 19981999.

4 Reppel É., "Les armuriers à Tours au XVIe siècle », mémoire de maîtrise de l'Université de Tours, CESR, sous la direction de Pascal Brioist, 2002-2003.

5 Du Bellay M., Les mémoires de Mess. Martin Du Bellay, seigneur de Langey, Paris, Chez Abel l'Angelier, libraire juré, 1582 ; Tremoille L. de, Les La Trémoille pendant cinq siècles, tomes 2 et 3, Nantes, É. Grimaud, Imprimeur-Éditeur, 1892.

6 Pfaffenbichler M., Armourers, Londres, British Museum Press, 1992, p. 23.
Fig. 1. La dynastie Merveilles. Les dates indiquées correspondent à la période d'activité attestée dans la ville. ? filiation inconnnue.

double interrogation en s'intéressant aux conditions de l'immigration, aux modes de diffusion de techniques nouvelles qui en résulte et, pour finir, aux processus d'intégration de ces armuriers étrangers.

\section{L'installation d'armuriers étrangers : une volonté royale}

L'installation d'une importante population d'armuriers étrangers à Tours procède dans une large mesure d'une volonté politique délibérée qui a engendré une dynamique faisant de Tours un pôle armurier de premier plan.

Les cinquante-neuf armuriers répertoriés sont originaires de pays limitrophes du royaume, pour une majorité de la péninsule italienne. Ceux-ci sont tous originaires de Lombardie, dans une aire comprise entre le lac Majeur, le lac de Côme et la ville de Milan. Ce qui ne relève pas du hasard. II s'agit là d'un des plus prestigieux centres de production d'armures de qualité du temps avec des maîtres armuriers renommés dans toute I'Europe. C'est ainsi que trois des dix grands noms de I'armurerie lombarde, parmi les plus réputés en Europe, sont représentés à Tours : la famille des Meraviglia, celle des de Lacques et celle des Daussonne ${ }^{7}$.

7 Pfaffenbichler M., Armourers, Londres, British Museum Press, 1992, p. 23 


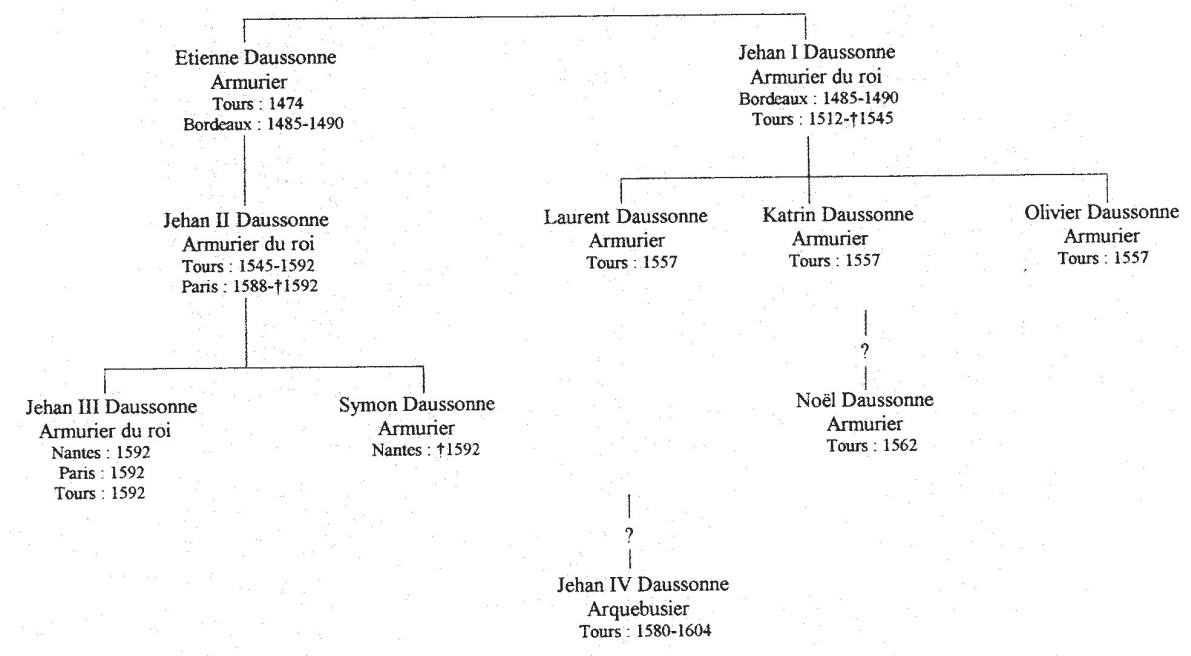

Fig. 2. La dynastie Daussonne. Les dates indiquées correspondent à la période d'activité attestée dans la ville. ? filiation inconnnue.

La famille milanaise Meraviglia, dont le nom fut francisé en Merveilles, semble s'être installée sur les bords de Loire dès 1425, année où Jean Merveilles s'y établit. Au xvi siècle, trois membres y sont encore présents : Jacques, Pierre et Loys, très actifs jusqu'en 1551, date à laquelle cette branche semble s'être éteinte avec la mort de Loys, les textes ne faisant plus mention de ce nom par la suite ${ }^{8}$. Toutefois, deux membres portant le même patronyme sont représentés à Paris avec Henri, décédé en 1568, et Jehan, dont l'activité est attestée en $1586^{\circ}$. Une présence à Tours qui a duré près de cent ans fait des Merveilles une des plus importantes dynasties d'armuriers tourangeaux (cf. fig. 1).

La famille de Lacques, originaire comme son nom I'indique de Lecco, près de Milan, est, elle, représentée dans la cité ligérienne depuis 1497. La famille Daussonne, présente à Tours depuis 1474, est la plus importante des dynasties d'armuriers ayant exercé dans cette ville. Neuf Daussonne y ont été recensés, dont huit pour le seul Xvie siècle. La présence de certains membres de cette famille est aussi attestée à Paris, Nantes et Bordeaux. Le nom de Daussonne renvoie encore une fois au foyer originel de la famille, Osogna en Lombardie (cf. fig. 2).

En dehors de ces trois principales familles, d'autres armuriers originaires du nord de la péninsule italienne

8 Archives Départementales d'Indre et Loire [ensuite AD], 3E1/66/A, 10 juillet 1551, «Blanche Bougeault, veufve de feu Loys Merveilles, luy vivant armurier...».

9 Grodecki C., Documents du minutier central des notaires de Paris. Histoire de l'art au XV' siècle (1540-1600), tome II, Paris, Archives nationales, 1986, p. 45. exercent à Tours depuis le xve siècle. Ainsi les Prata, père et fils, ou encore Gabriel de Trez, fils de Balzarin, originaire de Trezzo, près de Lecco.

Si Louis XI, Louis XII et François Jer ont surtout favorisé l'implantation d'artisans italiens, le Saint-Empire Romain Germanique n'est pas en reste et ses armuriers sont également très convoités. Forts d'une tradition armurière de haute qualité, très innovateurs tant sur le plan technique que décoratif, les armuriers d'Allemagne du Sud et d'Autriche ont acquis une réputation d'excellence dans toute l'Europe. II n'est donc pas étonnant que des maîtres armuriers ou des apprentis d'origine germanique aient été vivement encouragés à venir s'installer dans le royaume. Peu présent à Tours au xve siècle, cet état évolue au cours du xvie siècle où les migrations de ces armuriers deviennent plus fréquentes.

C'est ainsi que Henri Bouthelanquenar, haubergeonnier originaire de Clèves, exerce à Tours dès $1471^{10}$. Hans de Pontgrah, natif de Nuremberg en Bavière, arrive en France en 1504 et se fixe à Tours où il se marie avec une tourangelle de souche. Au service de Louis x\| puis de François ler, il reçoit, en juin 1528, de la part de ce dernier, ses lettres de naturalisation ${ }^{11}$. Hans Berger, armurier du roi, lui aussi né à Nuremberg, apparaît pour la première fois à Tours en 1530 dans une minute du notaire Étienne Viau.

10 Chevalier B., La ville de Tours et la société tourangelle, 13561520, 2 tomes, Lille, Service de reproduction des Thèses-Université de Lille, 1974.

11 Archives nationales [ensuite AN], JJ 243, juin 1528, fo 132. 
Tout comme la Lombardie et les Terres d'Empire, les Pays-Bas méridionaux sont eux aussi représentés à Tours durant ces deux siècles. Là également, il s'agit d'une région d'Europe riche d'une forte tradition armurière avec des grands maîtres réputés, de dimension internationale. La cité ligérienne abrite donc, aux $x v^{e}$ et $x v l^{e}$ siècles, des artisans venus des trois zones de production d'armures les plus prestigieuses d'Europe.

Ces observations confirment les remarques de Claudine Billot qui classe en trois niveaux les pays ayant fourni à la France des spécialistes de la métallurgie et ce, dès le $x v^{e}$ siècle. Les pays les moins actifs seraient I'Angleterre et la Suisse qui ont délégué très peu d'artisans. Le Saint-Empire se situe à un niveau intermédiaire avec des armuriers, des orfèvres et des serruriers originaires de Nuremberg et du duché de Clèves. Ceuxci se sont installés en grand nombre en Touraine, en Bretagne, en Picardie et dans la région lyonnaise. Au même niveau se situe I'Espagne. On repère à Tours des armuriers valenciennois et dans le Roussillon des fondeurs de cloches catalans. Les Pays-Bas méridionaux et I'Italie du nord restent les grands pourvoyeurs d'une main-d'œuvre qualifiée ${ }^{12}$.

Dans quelles conditions cette immigration d'armuriers allogènes s'est-elle effectuée ? En dehors des conflits entre Louis XI et Charles le Téméraire qui sont à I'origine de la dispersion d'un grand nombre d'ouvriers spécialisés flamands, les migrations des artisans, principalement ceux de la métallurgie, ont été favorisées par les souverains, princes ou municipalités qui se disputaient leurs services. L'exemple des armuriers lombards est de ce point de vue significatif. Les rois de France reçoivent en présent, au $x v^{e}$ siècle, des armures de la part des ducs de Milan et font acheter des armures complètes en Italie ou auprès de revendeurs dispersés dans les grandes villes d'Europe. Lorsque Louis XI décide de créer à Tours le principal centre de production de harnois pour équiper son infanterie et ses gens d'armes, c'est tout naturellement qu'il demande à recruter des armuriers lombards, tant leur réputation n'est plus à faire. Des sommes importantes sont allouées aux artisans afin de les inciter à venir s'installer dans la capitale ligérienne. L'autre moyen d'attirer des talents consiste à promettre à l'artisan armurier d'être rattaché à la mai-

12 Billot $C_{1}$. «Métiers de la métallurgie et migrations en France à la fin du Moyen Age », dans Benoit P., Cailleaux D. éd., Hommes et travail du métal dans les villes médiévales, Paris, AEDH, 1988, pp. 227-239, p. 231. son du roi. Ce qui lui vaut de nombreuses prérogatives liées à ce statut.

Certains armuriers sont prêtés aux rois de France. Dans la lettre de naturalisation de Jacquemin Ayrolde, il est précisé que ce natif de Milan a été prêté par le duc Galeazzo Maria Sforza au roi Charles VII ${ }^{13}$. Prêté et non donné, tant il est vrai que ces artisans contribuaient pour beaucoup au prestige des ducs de Milan et ce, dès I'époque des Visconti. Cependant, face à I'hémorragie d'ouvriers qualifiés, les princes lombards finissent par mettre leur veto à cette émigration vers la France dès $1450^{14}$. Ils n'apprécient guère de voir partir des artisans propres à leur assurer une avance technologique sur leurs voisins, surtout dans un domaine aussi stratégique que I'armement. Claudine Billot cite un exemple similaire pour les Pays-Bas méridionaux : en 1515, la municipalité de Bruxelles ne laisse pas s'expatrier en Angleterre des armuriers sollicités par Henri VIII qui cherche à développer le centre armurier de Greenwich.

Cependant, les migrations de spécialistes transalpins de l'armurerie vers la France et en particulier la Touraine sont facilitées par les guerres d'Italie. Par exemple, le Milanais Loys de Lacques, sommelier des armures du roi, armurier chargé de l'entretien, de la réparation et de la bonne conservation des armures du monarque, arrive en France en 1497 au retour d'Italie de l'armée de Charles VIII. II met son art au service de ce souverain et de ses deux successeurs, Louis XII et François ler, lequel lui accorde ses lettres de naturalisation datées de 1528 en ces termes:

«Françoys, par la grâce de Dieu, roy de France, sçavoir faisons que nous avons receu l'humble supplication de nostre chier et bon ami Loys de Lacque, dit Merveilles, sommelier ordinaire de nostre armurerie, natif de la ville de Millan, contenant que trente ans a et plus, il est venu demourer et c'est tenu en nostre royaume et depuis led. temps retenu et continué, tant au service de feuz noz prédécesseurs les roys Charles huystiesme et Loys, notre beaupère dernier décédé, que Dieu absoille, que de nous, aud. estat, ouquel il s'est employé a son pouvoir jusqu'à présent. En quoy faisant, se sera marié et habitué en nostre ville de Tours et de luy et de feue sa femme décédée, seroit yssu enfans, dont les anciens sont aussi en nostre service, en semblable estat de nostre armurerie. Pourquy, en persistant a l'intencion qu'il a tousiours eue, a deslibéré finir aud lieu de Tours en une petite maison qu'il a par

13 Ibid., p. 233.

14 Ibid., p. 230. 
cy-devant acquise ou ailleur en nostre royaume. Pour ce, nous inclinant libéralement a la supplication et requeste dud. suppliant, avons a icelluy Loys de Lacques, dit Merveilles, donné et octroyé congé de demourer en nostre dict royaume et en icelluy, acquerir et posseder tous et chascun les biens tant meubles que immeubles qu'il y pourra licitement avoir.

Douné a St-Germain-en-Laye ou moys de décembre I'an de grâce mil Vc XXVII et de nostre règne le $X|I|^{\mathrm{e}}{ }^{15}$

Une fois bien établis dans le royaume avec une clientèle de qualité, ces artisans peuvent diffuser, en toute liberté semble t-il, leurs techniques et leur savoirfaire sur l'ensemble du territoire. Et la première manière de le faire passe tout d'abord par le biais de réseaux.

Les sources démontrent que les armuriers tourangeaux ont des rapports assez fréquents avec leurs homologues et ce, dans l'ensemble du royaume. Ils sont aussi en relation avec un ou plusieurs réseaux de fournisseurs, certainement restreints tant le métier est spécialisé. C'est ainsi que des familles d'armuriers tourangeaux d'origine transalpine, comme les Daussonne ou les Merveilles, ont des collaborateurs ou des parents qui exercent dans d'autres centres armuriers d'importance tels que Paris, Nantes, Bordeaux ou Lyon et sont en mesure de pouvoir s'échanger des informations. Cela est surtout vrai pour les armuriers du roi. On peut également se demander si les armuriers étrangers ont tissé des réseaux avec leur pays d'origine et leurs confrères y exerçant, facilitant ainsi la transmission des nouvelles techniques et des savoir-faire. Plusieurs indices dans les sources le laissent entendre et il n'est pas interdit de penser que les nouveautés techniques arrivent par ce biais. Martin du Bellay, dans ses mémoires, relate à ce propos un fait très intéressant et qui mérite réflexion ${ }^{16}$ : la mort d'un serviteur de l'écurie du roi surnommé « Merveilles » décapité sur ordre du duc de Milan pour cause d'espionnage au profit du roi François ler17. Beaucoup d'indices comme les dates, I'origine, les fonctions et même le surnom laissent à penser qu'il s'agit de Loys de Lacques, armurier et sommelier des armures du roi ${ }^{18}$. En effet ce «Merveilles», originaire

15 AN JJ 243, f¹58, décembre 1528.

16 Du Bellay M., op. cit. note 5.

17 Ibid., livre IV, p.196.

18 Le surnom de "Merveilles » qui ne doit pas être confondu avec la famille du même nom, est en effet systématiquement attribué à Loys de Lacques, aussi bien dans son acte de naturalisation (AN, JJ 243, fo 158) que dans les actes notariés (3E1/40, 12 février $\left.1523, f^{\circ} 254\right)$. du Milanais, arrive en France avec I'armée royale et se met au service de l'Écurie du roi, tout comme Loys de Lacques. En 1531, dit Du Bellay, « ledit Merveilles avoit demandé congé au roy pour aller en Lombardie visiter ses parents ». Quelque temps après, le roi le convoque à Fontainebleau pour lui remettre un titre d'ambassadeur auprès du duc de Milan. Merveilles accepte à I'unique condition que "ce tiltre d'ambassadeur fut et demeurast secret » et que sa présence auprès du duc soit « non comme ambassadeur mais comme sollicitant ses propres et particulières affaires. Ce que le roi lui accorda en lui donnant des lettres de créances. Il s'agit donc d'un armurier au service du souverain qui de surcroît dirige une affaire ou un commerce qui semble prospère puisqu'il a « ésté bien traité en France, de manière qu'il y estoit fort riche » et peut alors se rendre en Lombardie en « gros équipage, accompagné de serviteurs ». Cet avantage correspond parfaitement aux prérogatives des armuriers du roi. Enfin, les dates d'apparition et de disparition de cet individu coïncident avec celles de Loys de Lacques dans les sources.

S'il s'agit bien de lui, l'anecdote prouve d'une part que les armuriers peuvent parcourir l'Europe pour affaires, ce qui démontre l'existence de réseaux actifs, et d'autre part que certains de ces artisans au service du souverain ont des rapports vraiment privilégiés avec ce dernier qui peut leur confier des missions capitales pour les affaires du royaume, en l'occurrence le Milanais, si cher au yeux du roi.

Cette approche des réseaux d'armuriers demeure succincte et certains points méritent d'être approfondis. Étudier les correspondances privées qui nous sont parvenues, comme celle de la famille milanaise Negroli, afin d'y trouver des parallèles avec l'armurerie tourangelle ou tenter de relever dans les archives de grands centres armuriers français, mais surtout européens, des noms d'armuriers ayant exercé à Tours serait vraiment d'une grande utilité pour reconstituer en partie certains réseaux, aussi complexes soient-ils. Cependant, plus que les voyages et les contacts ponctuels, les principaux vecteurs de la diffusion des techniques restent avant tout chez les armuriers l'apprentissage et le compagnonnage.

Un constat initial s'impose. La diffusion des techniques italiennes ou germaniques se fait par le biais de maîtres français ayant été compagnons chez des artisans italiens ou allemands. À Tours, des maîtres transalpins, comme Balzarin de Tretz, Jacques Merveilles ou encore Jehan Daussonne, emploient de nombreux apprentis et compagnons, pour la plupart régnicoles, 


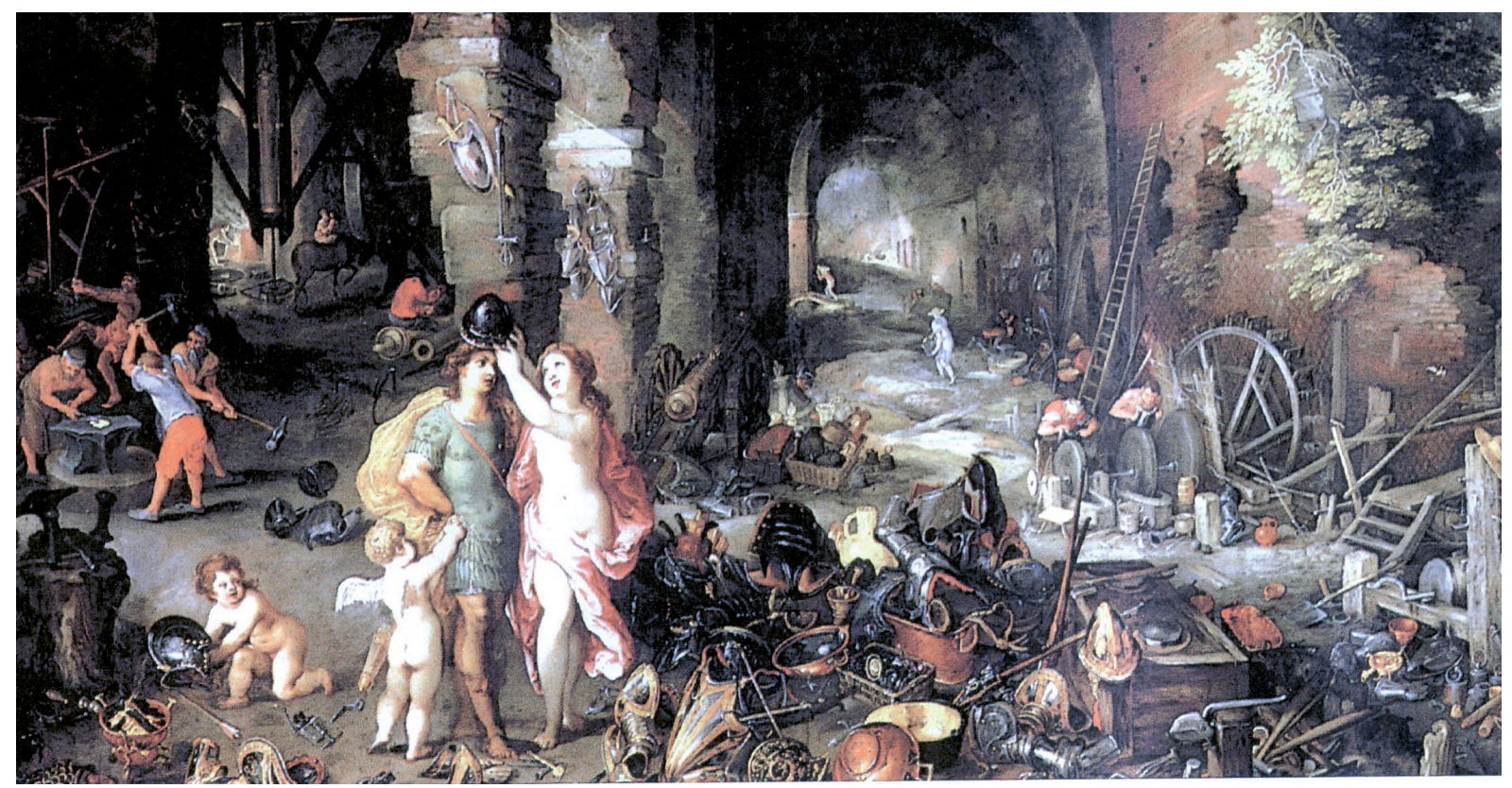

Fig. 3 Jan Breughel le jeune et Hendrik Van Balen, Les quatre éléments: le feu. Musée des Beaux-arts de Lyon.

qui, une fois devenus maîtres à leur tour, mettent en œuvre les techniques apprises. Cela est visible dans les commandes de sous-traitance. C'est de cette manière que des secrets de fabrication jalousement gardés se transmettent entre un maître et son apprenti favori. Cela est également valable pour les autres grands centres armuriers du pays. D'où la diffusion dans tout le royaume d'une École italienne, avec un style artistique et un savoir-faire propre.

\section{La diffusion d'un savoir-faire unique}

L'usage croissant du grotesque par les décorateurs d'armures se trouve lié à la diffusion, depuis I'Italie au reste de l'Europe, de livres d'emblèmes. Le goût de l'époque est au symbolisme et à l'allégorie. On retrouve parfois chez les armuriers des livres de patrons comme à Amiens.

Au milieu du xvl ${ }^{e}$ siècle, le roi Henri II favorise cette tradition d'emprunt en encourageant l'émergence d'un nouveau style : le maniérisme. Ce souverain a en effet incité les armuriers du royaume à s'inspirer de l'École de Fontainebleau sous l'égide d'Étienne Delaune pour I'ornementation des armures. Figures allégoriques et mythologiques peuplent les plastrons et autres pièces de harnois. L'armurier tourangeau Jehan II Daussonne qui vient travailler à Paris à l'invitation d'Henri II, com- me beaucoup de ses confrères tourangeaux, semble avoir été influencé par la mode maniériste. Installé rue de la Heaumerie, près de Saint-Germain-l'Auxerrois, il réalise en avril 1588 « un harnois complect, le tout d'or moulu et gravé et esmaillé, led. émail sur l'or de la plus belle et riche façon » destiné à Joseph de La Mothe, gentilhomme de la Chambre ordinaire du roi ${ }^{19}$. Il réalise ainsi des harnois niellés, dorés et embossés dans le même style que la célèbre armure dite de « Mars à la victoire » datée de 1570 environ et visible au musée de I'Armée de Paris.

La technique de la gravure en creux au moyen de l'eau-forte, qui n'est autre que de l'acide nitrique, constitue un autre exemple de technique de décoration utilisée par les Italiens dès la fin du Moyen Âge et qui se diffuse progressivement en France. Elle connaît au $x \mathrm{~V}^{\mathrm{e}}$ siècle un regain dû aux progrès de la gravure sur bois et sur cuivre en Allemagne du Sud, notamment grâce aux travaux du peintre et graveur Albrecht Dürer.

Cette diffusion de la mode italienne n'est pas spécifique à la France. Elle se retrouve dans une grande partie de l'Europe, particulièrement en Angleterre où I'arsenal de Greenwich produit ce type d'armure et aussi dans les Pays-Bas méridionaux comme par exemple à Liège ou à Bruxelles. Dans le tableau le Feu, tiré de la série des Quatre éléments, Jan Breughel le Jeune et Hendrik Van Balen représentent des pièces de harnois de formes et d'ornements d'inspiration transalpines, alors en vogue (cf. fig. 3)

19 Grodecki, C., op. cit. p. 281. 
Les migrations d'artisans hautement spécialisés et leurs enseignements demeurent donc les principaux vecteurs de diffusion de nouvelles techniques d'armurerie.

Comme nous l'avons constaté, ceux-ci se sont installés en France, en premier lieu grâce à leur savoir-faire, et encouragés par les Valois. II s'agit à présent de cerner les processus d'intégration de ces armuriers halogènes au sein du royaume et plus particulièrement à Tours.

\section{Des artisans parfaitement intégrés}

Dans la pratique quotidienne, ces artisans étrangers sont rapidement assimilés et ce, dès la première génération et connaissent une réussite sociale incontestable. Si, au $x v^{\text {e }}$ siècle, la production tourangelle est surtout une fabrication de grande série destinée à équiper les armées royales, Tours devient cependant, dès le Xvie siècle, le centre d'une production de harnois d'une qualité nettement supérieure. Par leur savoir-faire et la qualité de leurs créations, les armuriers tourangeaux d'origine étrangère, germanique et surtout italienne, intègrent rapidement le cercle restreint des fournisseurs attitrés des souverains Valois et des Grands du royaume ${ }^{20}$.

Ainsi, Loys Merveilles réalise en 1539, avec trois autres armuriers, pour le compte de François ler des harnois destinés au cardinal de Lorraine, au duc de Guise et à Anne de Montmorency ${ }^{21}$. En dehors des besoins d'ordre purement militaire, d'autres types d'armures, aux caractéristiques mécaniques différentes, font la réputation de Tours et de ses armuriers, en particulier de Jacques Meraviglia, dit Merveilles. II s'agit des armures destinées aux tournois et aux joutes, exercices encore fort prisés au Xvl ${ }^{e}$ siècle. Si la Lombardie et l'Autriche sont réputées pour la fabrication de ce type de protection, c'est bien à Tours que le duc de Valois, François d'Angoulême, se fait confectionner plusieurs harnois de joutes moins d'un an avant son accession au trône de France.

II s'agissait alors de participer à un grand tournois donné à Paris à l'occasion des cérémonies du mariage de Louis XII et de Marie d'Angleterre en novembre 1514. François, comte d'Angoulême et duc de Valois, afin d'y briller, se commande à Tours un ensemble de pièces d'armures dont le coût s'élève à un montant total de quatre cent quatre-vingt-douze livres et cinq sols tournois ${ }^{22}$. II se trouve qu'une fois encore c'est un

20 Reppel, É. op. cit.

21 Catalogue des actes de François Ier, Paris, Imprimerie nationale, 1907, t. 8, p. 198.

22 AN, KK 240. Compte de l'écurie de François ler.
Italien, Jacques Merveilles, qui reçoit la commande. La conception de harnois aussi complets a exigé de Jacques Merveilles et de ses compagnons beaucoup de soins et de temps puisque quinze journées ont été nécessaires rien que pour prendre les mesures du prince et deux mois et demi ont été consacrés à leur réalisation. À cela s'ajoutent de nombreux allers et retours entre Tours et Paris, en passant par Étampes où résidait François, afin de réaliser les essayages. Jacques Merveilles a eu aussi le privilège d'assister aux tournois, peut-être pour revêtir lui même de ses harnois son prestigieux client $^{23}$.

Cette spécialisation assure la position sociale et financière de ces artisans. Les armuriers tourangeaux $d$ 'origine étrangère s'intègrent à cette époque aux plus riches marchands et artisans de la ville.

La venue d'artisans natifs de pays étrangers ne semble par ailleurs pas avoir provoqué de conflits ni de phénomènes de rejet de la part de la population tourangelle, du moins au regard des archives. Aucune discrimination spatiale au sein de la ville n'apparaît. $\mathrm{Ni}$ les armuriers d'origine italienne ni même ceux d'autres contrées ne semblent avoir été exclus de la vie locale. Au contraire, Jacques Merveilles, maître juré de sa corporation, représente régulièrement, en plus des armuriers, les habitants de sa paroisse aux assemblées municipales ${ }^{24}$. II est vrai aussi que de nombreuses mesures officielles, émanant du roi, ont favorisé l'intégration de ces artisans étrangers. La trajectoire sociale ascensionnelle débute souvent par l'attribution d'un statut favorable, comme devenir armurier du roi.

Constituant une classe à part dans la profession, avec des fonctions propres et des prérogatives qui s'y rattachent, les armuriers et brigandiniers du roi ne bénéficient donc pas que d'un simple titre honorifique. Ils sont tous officiers de l'Hôtel du roi, rattachés en particulier à son écurie. Ils habitent en ville et ne sont astreints en aucun cas à l'obligation de résidence à la $\operatorname{cour}^{25}$. Ce statut peut même leur être accordé à vie étant donné que certains d'entre eux ont été employés par plusieurs souverains successifs. Ces artisans touchent de la part du souverain des gages très élevés. Bernard Chevalier rapporte que, sous Charles VIII, un armurier attaché à la cour pouvait se voir attribuer une

23 II est en effet précisé sur ce document...

24 AM Tours, BBR 16, 7 avril 1522 et BBR 19, 14 juillet 1527, $\mathrm{F}^{\circ} 74$.

25 Warnke, M., L'artiste et la cour, aux origines de l'artiste moderne, Paris, Éd. de la Maison des Sciences de l'Homme, 1989, p. 88. 
rémunération susceptible de s'élever à deux cent quarante livres par $a^{26}$. Ils perçoivent en plus une rémunération pour chaque réalisation ponctuelle. C'est le cas de Loys de Lacques qui touche quatre livres tournois pour avoir apporté des modifications à deux cuirasses appartenant au roi Louis XII27. Ces traitements leur garantissent donc un confort matériel indéniable. II arrive fréquemment qu'un artisan ou un artiste de cour voie ses revenus s'accroître par la transformation de son salaire en commission à vie ${ }^{28}$. Cette commission peut se présenter sous des formes diverses : rentes sur des biens, des droits financiers, des donations de terre ou de bénéfices, transmissibles aux héritiers du bénéficiaire. C'est ainsi que François I $^{\text {er }}$ fait don aux enfants de Jehan de Lacques des bénéfices de leur père après son décès ${ }^{29}$. De plus, être fournisseur de la cour leur apporte une grande notoriété et un certain prestige. Jehan I Daussonne, lorsqu'il signe un acte notarié, n'oublie jamais de rappeler son statut (cf. fig. 4).

Ce n'est donc pas un hasard si une certaine clientèle s'approvisionne chez ces artisans. II n'existe en effet aucune exclusivité et les armuriers royaux reçoivent et exécutent de nombreuses commandes privées ${ }^{30}$. Il ne fait donc aucun doute que les armuriers et brigandiniers bénéficiant de ce statut sont privilégiés par rapport aux autres armuriers de la ville et sont certainement très enviés.

La deuxième étape implique généralement un mariage avec une Tourangelle de souche qui achève le processus d'intégration : Jehan I Daussonne, par exemple, s'est marié avec la fille d'un des libraires du roi. La fille de Gabriel de Tretz, Jehanne, épouse Nicole Charretier, maire de Tours. Les brigandiniers, aux revenus plus modestes, convolent en général avec des filles issues de familles de marchands.

Certains armuriers bien intégrés participent activement à la vie politique de la cité et certains d'entres eux sont même devenus maire ou échevin. Au xve siècle, sur sept armuriers élus à l'échevinage, cinq individus sont des halogènes. C'est le cas par exemple des Italiens Balsarin de Tretz et Jehan Roquenert ${ }^{31}$. La désignation comme échevin de Tours autorise I'anoblissement. La dernière

26 Chevalier, B., "Tours, ville royale», op. cit. p. 260.

27 CHAN KK 86, Compte de l'écurie de Louis XII,1508, F 53.

28 Warnke, M., op. cit., p. 163.

29 Cf. Catalogue des actes de François Ier, Paris, Imprimerie nationale, 1907, t. 7, p. 535.

30 Les exemples sont nombreux dans les actes notariés.

31 Painsonneau, S. op. cit., p. 233

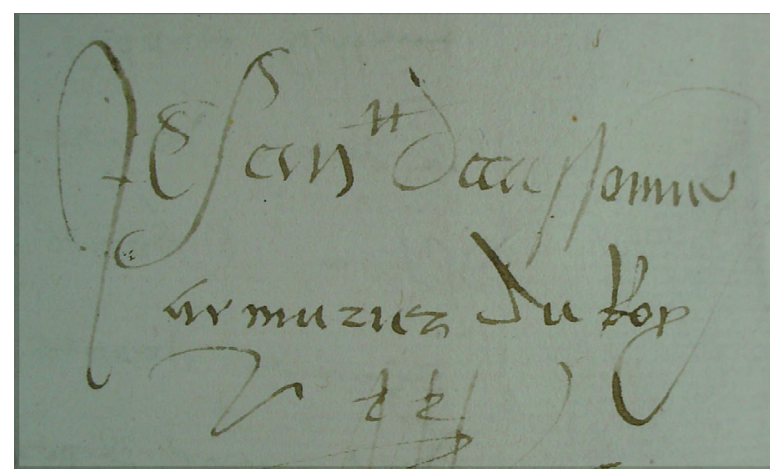

Fig. 4. Signature de Jehan Daussonne, armurier du roi AD d'Indre et Loire, 3E1/55, le 8 avril 1541

étape est marquée par les lettres de naturalisation qui achèvent juridiquement cette assimilation sociale.

\section{Conclusion}

Les spécialistes de l'armurerie défensive exerçant à Tours, d'origine étrangère, principalement italienne, dépositaires d'une longue expérience et d'un savoirfaire unique en Europe, ont contribué en grande partie à la richesse et à la réputation de la cité ligérienne dans le domaine de l'armurerie dès le milieu du xve et durant tout le $x v l^{e}$ siècle. Favorisés par le pouvoir royal, qui les a encouragés à venir s'installer dans le royaume, ces artisans pérégrins se sont très bien assimilés, au point qu'une grande majorité d'entre eux, dont certains installés en Touraine dès le $x v^{\mathrm{e}}$ siècle, ont fait souche et sont à l'origine de véritables dynasties, à l'exemple des familles Merveilles et D'Aussonne. Certaines d'entre elles sont encore en activité au XvII siècle, comme l'attestent les sources. Cependant Tours ne fait pas exception. Des armuriers de renoms transalpins ou encore originaires des terres d'Empire, se retrouvent aussi en grand nombre à Lyon et à Bordeaux. Toutefois, la renommée de Tours sera de courte durée. L'étape ultime est de quitter la capitale ligérienne en déclin pour rejoindre Paris où, dès la seconde moitié $d u x v l^{e}$ siècle, les plus fameux artisans tourangeaux d'origine étrangère de la seconde ou de la troisième génération sont invités par le roi à venir s'installer auprès d'autres grands maîtres italiens comme les Milanais Giovanni Pietro et Giovanni Ambrogio Negroli ou le Brescian Antoine Rigauti qui exercent rue de la Heaumerie.

Les armuriers italiens ne se sont pas seulement exportés en France. On les retrouve en grand nombre à Londres, en Flandres et dans les terres d'Empire participant, au travers de leurs pérégrinations, à la transmission des savoir-faire dans l'Europe de la Renaissance. 